\title{
Successful combination chemotherapy for metastatic inflammatory myofibroblastic tumor: A case report
}

\author{
KYOKO INADOMI $^{1}$, HOZUMI KUMAGAI ${ }^{1}$, KOTOE TAKAYOSHI ${ }^{1}$, HIROSHI ARIYAMA ${ }^{1}$, HITOSHI KUSABA ${ }^{1}$, \\ AKIHIRO NISHIE ${ }^{2}$, HIDETAKA YAMAMOTO ${ }^{3}$, KEN TAKASE ${ }^{4}$, MAMORU TANAKA ${ }^{1}$, KOSUKE SAGARA ${ }^{1}$, \\ YUTA OKUMURA $^{1}$, KENTA NIO $^{1}$, MICHITAKA NAKANO $^{1}$, SHUJI ARITA $^{1}$, \\ YOSHINAO ODA ${ }^{3}$, KOICHI AKASHI ${ }^{1}$ and EISHI BABA ${ }^{5}$ \\ ${ }^{1}$ Department of Hematology and Oncology, Kyushu University Hospital; Departments of ${ }^{2}$ Clinical Radiology and \\ ${ }^{3}$ Anatomic Pathology, Pathological Sciences, Graduate School of Medical Sciences, Kyushu University, Fukuoka 812-8582; \\ ${ }^{4}$ Department of Hematology, National Hospital Organization Kyushu Medical Center, Fukuoka 810-0065; ${ }^{5}$ Department of \\ Comprehensive Clinical Oncology, Faculty of Medical Sciences, Kyushu University, Fukuoka 812-8582, Japan
}

Received September 4, 2014; Accepted May 29, 2015

DOI: $10.3892 / 01.2015 .3708$

\begin{abstract}
A 64-year-old male presented with increased abdominal fullness and fever. Radiological examination revealed moderate ascites, a tumor with a diameter of $12.5 \mathrm{~cm}$ in the mesenteric region, as well as multiple tumors in the thoracic and abdominal para-aortic regions and in the left supraclavicular regions. Pathohistological findings of the biopsy specimen revealed atypical spindle cells accompanied by infiltration of lymphocytes. The plasmacytes were positive for CD68, murine double minute 2 and S-100, while they were negative for $\alpha$-smooth muscle actin, cyclin-dependent kinase 4 and anaplastic lymphoma kinase. Clinically, the patient presented systemic symptoms and laboratory results indicated an elevation in the inflammatory response, while the CT and MRI findings were consistent with an inflammatory myofibroblastic tumor (IMT). Based on the clinical and histological findings, the patient was diagnosed with IMT. In total, 4 cycles of combination chemotherapy with doxorubicin and ifosfamide were administered. Tumor size reduction by $50 \%$ was achieved subsequent to the 4 th chemotherapy cycle. In conclusion, successful control of this rare metastatic IMT was achieved by systemic chemotherapy.
\end{abstract}

Correspondence to: Professor Eishi Baba, Department of Comprehensive Clinical Oncology, Faculty of Medical Sciences, Kyushu University, 3-1-1 Maidashi, Higashi-ku, Fukuoka 812-8582, Japan

E-mail: e-baba@intmed1.med.kyushu-u.ac.jp

Key words: inflammatory pseudotumor, metastatic inflammatory myofibroblastic tumor, chemotherapy, anaplastic lymphoma kinase rearrangement negative

\section{Introduction}

Inflammatory myofibroblastic tumor (IMT) is a relatively rare disease that predominantly occurs in the peritoneum, retroperitoneum and lungs of young people; a previous study of 38 cases reported a median age of 8.5 years (1). This disease is histologically characterized by the proliferation of various spindle cells, which are of mesenchymal cell origin, in myxoid or collagenous stroma with prominent inflammatory infiltrates (2). IMT cells can potentially behave as locally invasive and are rarely metastatic (in $<5 \%$ of cases) (3). In recent studies, a chromosomal translocation involving $2 \mathrm{p} 23$ and subsequent anaplastic lymphoma kinase $(A L K)$ gene rearrangement was identified in $\sim 50 \%$ of IMT cases $(3,4)$. The ALK inhibitor, crizotinib, was demonstrated to be an effective therapy for $A L K$ rearrangement-positive IMT cases.

Complete surgical resection is typically conducted for localized IMTs (5); however, no standard therapeutic strategy has been established for inoperable or metastatic IMTs. In the present study, an extremely rare case of metastatic IMT that responded successfully to combination chemotherapy of doxorubicin and ifosfamide was reported.

\section{Case report}

A 64-year-old male presented with abdominal fullness, fever and severe fatigue in March 2014. These symptoms had worsened within half a month. Following a visit to a primary care doctor in March 2014, a computed tomography (CT) scan revealed a moderate volume of ascites and a mass in the middle of the abdomen (Fig. 1A). Initially, lymphoma was suspected, and oral prednisolone (60 mg/day) was administered for 3 days as a therapy with diagnostic intent; however, no relief of the symptoms was observed. Exploratory laparotomy indicated a protruded large tumor in the mesenterium and 2,500 $\mathrm{ml}$ of chylous ascites. Microscopic examination of the tumor biopsy sample was unable to establish a diagnosis. In April 2014, the patient was referred to the Department of Hematology and 

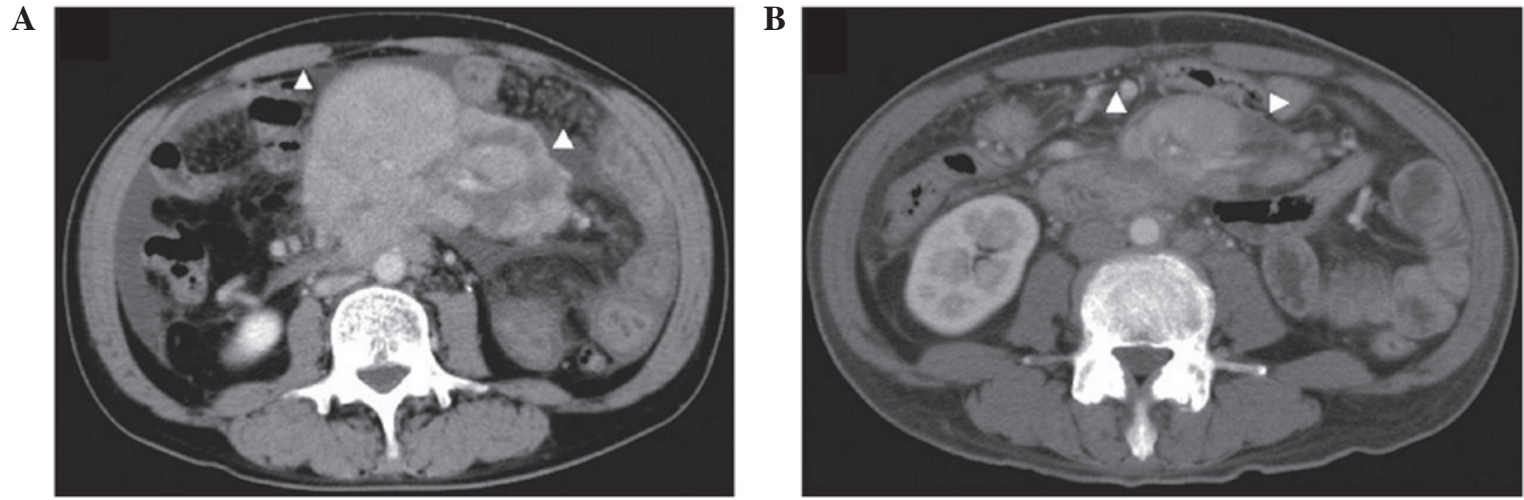

Figure 1. Enhanced CT images prior to and following chemotherapy. (A) CT scan prior to chemotherapy revealed a lobulated mass with patchy enhancement $(12.5 \times 8.7 \mathrm{~cm})$ that surrounded the mesenteric vessels (arrow heads) and a moderate volume of ascites in the abdominal cavity. (B) Following the 4th cycle of chemotherapy, the mass was reduced to a size of $6.2 \times 3.6 \mathrm{~cm}$ and contrast enhancement was also reduced. No ascites were identified in the abdominal cavity.

Oncology at Kyushu University Hospital (Fukuoka, Japan) for further evaluation. At the time of admission, the Eastern Cooperative Oncology Group performance status (6) had improved from grade 3 to grade 1 following removal of the ascites, and the patient suffered from low back pain, fatigue and fever. The laboratory results were as follows: C-reactive protein, $9.25 \mathrm{mg} / \mathrm{dl}$ (normal, $\leq 0.14 \mathrm{mg} / \mathrm{dl}$ ); erythrocyte sedimentation, $105 \mathrm{~mm} / \mathrm{h}$ (normal, $2-10 \mathrm{~mm} / \mathrm{h}$ ); platelet count, $45.6 \times 10^{4} / \mu \mathrm{l}$ [normal, (158-348)x103/pl]; interleukin-6 (IL-6) level, $140 \mathrm{pg} / \mathrm{ml}$ (normal, $\leq 4.0 \mathrm{pg} / \mathrm{ml}$ ). Fluorodeoxyglucose-positron emission tomography (FDG-PET)/CT revealed hypermetabolic masses in the mesenteric region [maximum standardized uptake value $\left.\left(\mathrm{SUV}_{\text {max }}\right), 23.2\right]$, as well as in the celiac artery and para-aortic region $\left(\mathrm{SUV}_{\max }, 18.5\right)$, the dorsal region of the thoracic descending aorta $\left(\mathrm{SUV}_{\max }, 7.4\right)$ and the left supraclavicular region $\left(\mathrm{SUV}_{\max }, 5.8\right.$; Fig. 2). Magnetic resonance imaging (MRI) examination indicated tumors with mixed high- and low-intensity signals in a T2-weighted image (T2-WI).

Intensive histological examination of the previously obtained tumor sample demonstrated the proliferation of mildly atypical cells that possessed enlarged, spindle to polygonal shaped nuclei and vesicular chromatin arranged in a haphazard pattern. These tumor cells were accompanied by an infiltration of lymphocytes and plasmacytes (Fig. 3A). Immunohistochemical staining of the atypical tumor cells was categorized as follows: Positive, $>10 \%$ cells stained, moderate-high intensity; negative, $0 \%$ cells stained; or focally positive, not within the criteria for positive or negative. The analyses revealed that the cells were positive for CD68, focally and weakly positive for murine double minute 2 (MDM2; Fig. 3B) and focally positive for S-100 protein; by contrast, the cells were negative for $\alpha$-smooth muscle actin, cyclin-dependent kinase 4 (CDK4), ALK and CD35. The tumor was also negative for Epstein-Barr virus-encoded small RNAs as assessed by in situ hybridization. In addition, the plasmacytes were negative for immunoglobulin G4 (IgG4). These histological features suggested a type of inflammatory pseudotumor (IPT)-like lesion; however, establishing a more accurate diagnosis was difficult. The differential diagnosis included IMT, IPT-like dedifferentiated liposarcoma (DLS).

At 7 days after admission, epigastrium and back pain, a high fever and significant elevation of serum pancreatic and biliary enzyme levels [lipase, $712 \mathrm{U} / 1$ (normal, 16-51 U/l);

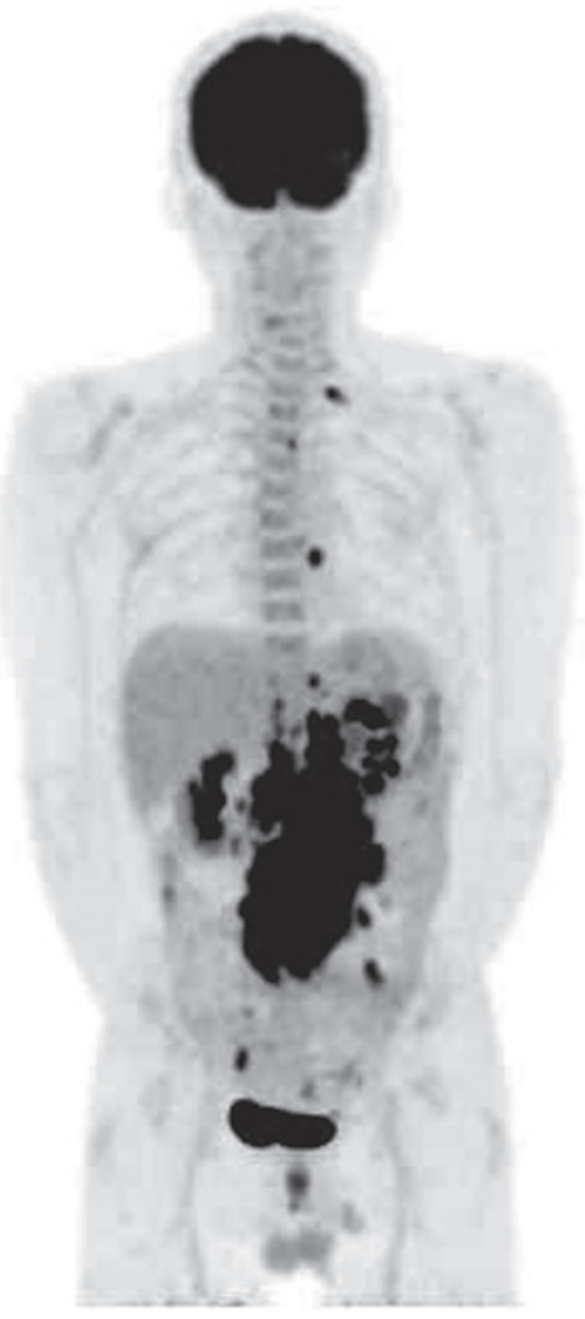

Figure 2. Fluorodeoxyglucose-positron emission tomography image, which revealed hypermetabolic masses in the region of the mesentery $\left(\mathrm{SUV}_{\max }, 23.2\right)$, along the celiac artery and the para-aortic region $\left(\mathrm{SUV}_{\max }, 18.5\right)$, in the dorsal region of the thoracic descending aorta $\left(\mathrm{SUV}_{\max }, 7.4\right)$ and in the left supraclavicular region $\left(\mathrm{SUV}_{\max }, 5.8\right)$. $\mathrm{SUV}_{\max }$, maximum standardized uptake value.

total bilirubin, $3.5 \mathrm{mg} / \mathrm{dl}$ (normal, $0.4-1.5 \mathrm{mg} / \mathrm{dl}$ ); alkaline phosphatase, 1,553 U/l (normal, 106-322 U/l); $\gamma$-glutamyl transpeptidase, 1,070 U/1 (normal, 13-64 U/1)] were manifested. An MRI scan revealed tumor invasion of the pancreas 

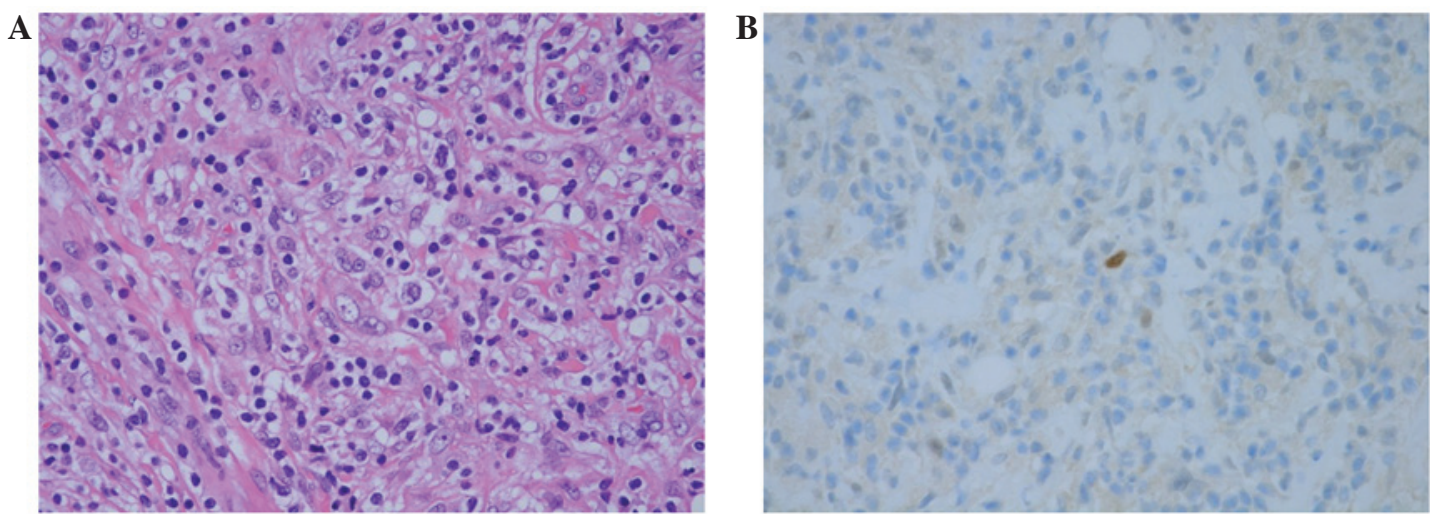

Figure 3. Pathohistological examination of the biopsy sample. (A) Proliferation of atypical cells that display enlarged and spindle to polygonal shaped nuclei and vesicular chromatin arranged in a haphazard pattern is shown. Tumor cells were accompanied by infiltration of lymphocytes, plasmacytes, focally aggregated epithelioid histiocytes and multinucleated giant cells. Mitotic figures are not conspicuous. Hematoxylin and eosin staining; magnification, $x 400$. (B) Immunohistochemically, the atypical tumor cells were focally and weakly positive for MDM2. MDM2 staining; magnification, $\mathrm{x} 400$. MDM2, murine double minute 2.

head causing severe stenosis of the main pancreatic and choledochal duct. Catheterization was then performed and the tumor exhibited rapid growth and invaded to the adjacent tissue, which suggested a malignant neoplasm.

Although a diagnosis could not be confirmed based on the histological features alone, the patient was finally diagnosed with an IMT based on the aforementioned clinical and histological findings, including inflammatory symptoms and radiological images. The patient received doxorubicin (15-25 mg/m $\mathrm{m}^{2}$, days 1 and 2) and ifosfamide $\left(1.75-2 \mathrm{~g} / \mathrm{m}^{2}\right.$, days 1-5) every 3-4 weeks (AI therapy), beginning in May 2014. A reduced dosage of chemotherapy was used due to liver dysfunction and to avoid a fatal adverse event, such as febrile neutropenia, in the subsequent cycle. Following the 4th cycle of chemotherapy, a CT scan revealed a 50\% reduction in the baseline sum of the longest diameter of the target regions (from $12.5 \mathrm{~cm}$ to $6.2 \mathrm{~cm}$ ), no size changes of the masses (all 6-7 $\mathrm{mm}$ in the minor axis; these had been hypermetabolic on previous FDG-PET in the celiac artery and para-aortic region, the dorsal region of the thoracic descending aorta and the left supraclavicular region), the disappearance of ascites and the absence of new lesions, which was classified as partial response according to the Response Evaluation Criteria in Solid Tumors (7) (Fig. 1B). The patient experienced neutropenia (grade 4; according to Common Terminology Criteria for Adverse Events version 4.0) (8), febrile neutropenia (grade 3) and sepsis by Enterococcus cloacae (grade 4) during the 1st cycle, as well as anorexia (grade 2) and fatigue (grade 1) for 5-6 days following the initiation of each cycle of treatment. In addition, the patient did not present any tumor-associated symptoms and the laboratory data, including C-reactive protein level, erythrocyte sedimentation, platelet count and IL-6 level, were within the normal limits. The patient was planned to continue the chemotherapy up to a maximum of 7 cycles due to the dose-limiting cardiac toxicity of doxorubicin.

Around the 5th cycle of AI therapy, radiological examination revealed a moderate amount of ascites without any change in the size of other lesions. From the 6th cycle, oral prednisolone $(30 \mathrm{mg} /$ day) was initiated in combination with the ongoing therapy, however, there was no change in the amount of ascites. Following the 7th cycle of AI therapy, multiple new liver metastatic lesions and hypermetabolic masses in the mediastinum, axilla and intraabdominal region were revealed by FDG-PET. For second-line chemotherapy, the patient was administered with a combination therapy of gemcitabine and docetaxel, commencing in November 2014, which was ineffective. The patient succumbed to liver failure at around 7 months after the start of AI therapy.

The patient provided written informed permission for the publication of this case.

\section{Discussion}

Inflammatory pseudotumors (IPTs) include a wide variety of tumors from benign to malignant neoplasms, such as the calcifying fibrous tumor, IgG4-associated inflammatory tumor, IPT-like follicular dendritic cell sarcoma, IMT-like DLS and IMT. Although it is occasionally difficult to histologically differentiate between such tumors, the nature of these diseases is diverse, particularly in terms of etiology (3). A chromosomal translocation involving 2p23 and subsequent $A L K$ gene rearrangement was previously identified in $\sim 50 \%$ of IMT cases; however, older patients with IMT tend to be negative for this translocation and gene rearrangement $(3,4)$. While detection of $A L K$ gene rearrangement in IPTs yields the primary evidence for the diagnosis of IMT, a definitive diagnosis is frequently difficult to achieve in cases of IMT without $A L K$ gene rearrangement (3). The present case exhibited the following pathohistological features that were characteristic of IPT: i) proliferation of mildly atypical spindle cells; ii) low frequency of mitotic figures; and iii) prominent infiltration of various inflammatory cells, including plasma cells and lymphocytes. In addition, the patient suffered from severe general fatigue and fever, and blood tests revealed a prominent elevation of the inflammatory response; these features are compatible with a diagnosis of IMT (1). CT and MRI scans demonstrated that the tumors originated from the soft tissue of the abdomen and presented a combination of high and low signal intensities on a T2-WI. The region of low signal intensity on the T2-WI was enhanced in the delayed 
phase of the CT scan, which indicates a fibrotic region, and this finding is also consistent with IMT.

The greatest differential diagnosis may be DLS, which rarely exhibits similar pathohistological characteristics with IMT (9). DLS is often diagnosed in older adults, as in the present case. Considering the heterogeneity of the tumor, DLS cannot be definitively excluded from the diagnosis due to the relatively small size of the biopsy sample. A recent immunohistochemical study of DLS samples revealed strong positive staining for MDM2 and CDK4 due to amplification of chromosome 12 q13-15 and the subsequent co-amplification of the MDM2 and $C D K 4$ genes (10). MDM2 gene amplification has also been detected in $27 \%$ of IMT cases (11). Therefore, the focally- and weakly-positive staining of MDM2 and the negative staining of CDK4 in the present case could suggest a diagnosis of DLS; however, this evidence could not confirm or exclude DLS. Considering both the clinical and pathohistological findings, the current patient was finally diagnosed with IMT.

The primary therapy for IMT is complete resection of the tumors (5). However, this therapy may not be suitable in the present case due to the large size of the tumor surrounding large vessels, as well as due to the presence of metastatic regions. Although the number of previous reports on IMT is limited, a small number of cases have been reported (5,12-16). A study reporting a case with $A L K$ gene rearrangement-positive IMT demonstrated the failure of AI therapy followed by maintenance with imatinib subsequent to surgery (12). Another case of retroperitoneal IMT underwent systemic chemotherapy with vincristine plus methotrexate and radiation, and no recurrence was observed for 2 years (5). In addition, certain studies on IMT cases in children reported the use of various chemotherapeutic drugs or regimens, including methotrexate, vinblastine, cisplatin, doxorubicin and ifosfamide, which demonstrated variable responses and thus their efficacy is unclear $(13,17,18)$. Non-steroidal anti-inflammatory drugs (NSAIDs) and corticosteroids have also been studied as treatment options for IMT. Vascular endothelial growth factor (VEGF) and cyclooxygenase (COX)2 are highly expressed in the infiltrating inflammatory cells of IMT (14). The COX2 inhibitor is considered to suppress angiogenesis in the tumor tissue via interference with the COX2/prostaglandin/VEGF axis. Two abdominal IMT cases were reported to be responsive to NSAIDs (15). Systemic chemotherapy was administered for the present patient due to the effects of the aggressive tumor on the surrounding organs, including obstructive jaundice and chylous ascites. The combination chemotherapy of doxorubicin and ifosfamide that was employed in the current study was based on the chemotherapy regimen for unresectable non-small round cell soft tissue sarcomas (19). The 4th cycle of the chemotherapy induced $50 \%$ reduction in the tumor diameter, suggesting the effectiveness of the therapy. Celecoxib (400 mg per day) was also administered from the initiation of chemotherapy since the patient suffered from back pain, while dexamethasone (20 mg/day, days 1-6 of AI therapy) was used for prophylaxis of emesis. There is a possibility that these additional agents may have also affected the suppression of tumor growth.

The kinase activity of the ALK fusion protein has been suggested to play a pivotal role in $A L K$ rearrangement-positive IMT development, in a similar manner to that of the EML4-ALK fusion protein in non-small cell lung cancer and that of NPM1-ALK or other associated fusion proteins in aplastic large cell lymphomas (20). However, considering that only $\sim 50 \%$ of IMT cases present $A L K$ gene rearrangement and that cases with and without $A L K$ gene rearrangement exhibit almost the same clinical and pathohistological characteristics, it is possible that unknown gene alterations may exist in these IMT cells. Although IMT cells have slightly atypical cell morphology and are classified as intermediate malignant tumors, aggressive local invasion or distant metastasis are also observed. Further investigation of the tumorigenicity of $A L K$ rearrangement-negative IMT and establishment of effective therapies for these tumors is required. In conclusion, the current study reports a rare metastatic case of IMT in which disease control was achieved through systemic chemotherapy.

\section{References}

1. Meis JM and Enzinger FM: Inflammatory fibrosarcoma of the mesentery and retroperitoneum. A tumor closely simulating inflammatory pseudotumor. Am J Surg Pathol 15: 1146-1156, 1991.

2. Coffin CM, Watterson J,Priest JR and DehnerLP: Extrapulmonary inflammatory myofibroblastic tumor (inflammatory pseudotumor). A clinicopathologic and immunohistochemical study of 84 cases. Am J Surg Pathol 19: 859-872, 1995.

3. Gleason BC and Hornick JL: Inflammatory myofibroblastic tumors: Where we are now? J Clin Pathol 61: 428-437, 2008.

4. Lawrence B, Perez-Atayde A, Hibbard MK, Rubin BP, Dal Cin P, Pinkus JL, Pinkus GS, Xiao S, Yi ES, Fletcher CD, et al: TPM3-ALK and TPM4-ALK oncogenes in inflammatory myofibroblastic tumors. Am J Pathol 157: 377-384, 2000.

5. Kovach SJ, Fischer AC, Katzman PJ, Salloum RM, Ettinghausen SE, Madeb R and Koniaris LG: Inflammatory myofibroblastic tumors. J Surg Oncol 94: 385-391, 2006.

6. National Cancer Institute: Cancer Therapy Evaluation Program: Common Toxicity Criteria, version 2.0. March 1998. http://ctep. cancer.gov/protocolDevelopment/electronic_applications/docs/ ctcv20_4-30-992.pdf.

7. Eisenhauer EA, Therasse P, Bogaerts J, Schwartz LH, Sargent D, et al: New response evaluation criteria in solid tumours: Revised RECIST guideline (version 1.1). Eur J Cancer 45: 228-247, 2009.

8. U.S.Department of Health and Human Services; National Institutes of Health; National Cancer Institute: Common Terminology Criteria for Adverse Events(CTCAE) Version 4.0.May2009.http:// ctep.cancer.gov/protocolDevelopment/electronic_applications/ ctc.htm\#ctc_40.

9. Lucas DR, Shukla A, Thomas DG, Patel RM, Kubat AJ and McHugh JB: Dedifferentiated liposarcoma with inflammatory myofibroblastic tumor-like features. Am J Surg Pathol 34: 844-851, 2010.

10. Binh MB, Sastre-Garau X, Guillou L, de Pinieux G, Terrier P, Lagacé R, Aurias A, Hostein I and Coindre JM: MDM2 and CDK4 immunostainings are useful adjuncts in diagnosing well-differentiated and dedifferentiated liposarcoma subtypes: A comparative analysis of 559 soft tissue neoplasms with genetic data. Am J Surg Pathol 29: 1340-1347, 2005.

11. Yamamoto H, Oda Y, Saito T, Sakamoto A, Miyajima K, Tamiya S and Tsuneyoshi M: p53 Mutation and MDM2 amplification in inflammatory myofibroblastic tumors. Histopathology 42 : 431-439, 2003.

12. Butrynski JE, D'Adamo DR, Hornick JL, Dal Cin P, Antonescu CR, Jhanwar SC, Ladanyi M, Capelletti M, Rodig SJ, Ramaiya N, et al: Crizotinib in ALK-rearranged inflammatory myofibroblastic tumor. N Engl J Med 363: 1727-1733, 2010.

13. Bertocchini A, Lo Zupone C, Callea F, Gennari F, Serra A Monti L and de Ville de Goyet J: Unresectable multifocal omental and peritoneal inflammatory myofibroblastic tumor in a child: Revisiting the role of adjuvant therapy. J Pediatr Surg 46: E17-21, 2011.

14. Applebaum H, Kieran MW, Cripe TP, Coffin CM, Collins MH, Kaipainen A, Laforme A and Shamberger RC: The rationale for nonsteroidal anti-inflammatory drug therapy for inflammatory myofibroblastic tumors: A children's oncology group study. J Pediatr Surg 40: 999-1003, 2005. 
15. Przkora R, Bolder U, Schwarz S, Jauch KW, Spes J, Andreesen R and Mackensen A: Regression of nonresectable inflammatory myofibroblastic tumors after treatment with nonsteroidal anti-inflammatory drugs. Eur J Clin Invest 34: 320-321, 2004.

16. Kusunoki-Nakamoto F, Matsukawa T, Tanaka M, Miyagawa T, Yamamoto T, Shimizu J, Ikemura M, Shibahara J and Tsuji S: Successful treatment of an unresectable inflammatory myofibroblastic tumor of the frontal bone using a cyclooxygenase-2 inhibitor and methotrexate. Intern Med 52: 623-628, 2013.

17. Johnson K, Notrica DM, Carpentieri D, Jaroszewski D and Henry MM: Successful treatment of recurrent pediatric inflammatory myofibroblastic tumor in a single patient with a novel chemotherapeutic regimen containing celecoxib. J Pediatr Hematol Oncol 35: 414-416, 2013.
18. Favini F, Resti AG, Collini P, Casanova M, Meazza C, Trecate $G$ and Ferrari A: Inflammatory myofibroblastic tumor of the conjunctiva: response to chemotherapy with low-dose methotrexate and vinorelbine. Pediatr Blood Cancer 54: 483-485, 2010.

19. ESMO/European Sarcoma Network Working Group: Soft tissue and visceral sarcomas: ESMO Clinical Practice Guidelines for diagnosis, treatment and follow-up. Ann Oncol 25: iii102-iiil12, 2014.

20. Mano H: ALKoma: A cancer subtype with a shared target. Cancer Discov 6: 495-502, 2012. 